\title{
The Contrivance of Prism Rule-Based Algorithm using ADLs Dataset in Context Database Design
}

\author{
Shivakumar $\mathbf{C}^{1}$ and Siddhaling Urolagin ${ }^{2, *}$ \\ ${ }^{1}$ Computer Science Department, Jain University, Ramanagaram, Karnataka-562112, INDIA \\ ${ }^{2}$ Department of Computer Science, Birla Institute of Technology \& Science, Pilani, Dubai International Academic City, P. O. Box No. \\ 345055, Dubai, UAE
}

Received 2 October 2019; Accepted 20 March 2020

\begin{abstract}
The concept of context-awareness in the database is the contemporary issue which is under discussion and so much of work is in progress, but yet to achieve much progress in context reasoning and behavior adaptability. This research work mainly focuses on context data reasoning using Prism rule-based algorithm in which rules are been generated with the aid of ADLs dataset and context database design with the support of CEAR diagram, Context-aware use case diagram, context dimension tree. Queries are been executed in RDBMS environment by keeping context-awareness scenario in mind and tuple calculus queries are implemented. This research work briefs about query visualization where attribute value block has been introduced here. We have used ADLs dataset of Ordenza et.al [5] which was been captured by different sensors and devices within the living room with two users for 35 days. Our novel contribution is enactment of PRISM rule-based algorithm using ADLs data and instigated attribute value block in the query visualization part. This research work throughout perpetually portrays stratagem of prism rule-based algorithm of context database in ubiquitous computing environment designed for a specific application ADLs.
\end{abstract}

Keywords: Activities of Daily Living, Context Dimension Tree, Relational Database Management System, Structured Query Language

\section{Introduction}

Aging population has a considerable impact on health care system Zola, I.K et.al[12].Capturing the activities of daily living is useful to track the different activities performed by the aged peoples by the caregivers and accordingly they can decide their (active participation) actively doing some activities like leaving, toileting, showering, sleeping, breakfast, grooming, etc., in homely environment. Automatic health monitoring system ensures the safety and motivates the aged people to independently perform their tasks on their own and exposes the details to the concerned people [14]. Due to advancement in the health care system it has facilitated millions of people to live longer and safer by exploiting the sophisticated technology. Indeed, Medical experts believe that knowing the medical conditions at the earliest stage than paying heed at the critical stages by analyzing the changes in Activities of Daily Living (ADLs). It is Projected that by 2025 aged over 60 years will be around 158.7 million people in India $[13,14]$. It becomes the responsibility of any country to find a simple and easy way to eradicate such problems. One way of overcoming such problems is implementing smart health monitoring system in which daily activities of the old and disabled peoples can be identified by the help of pervasive systems. Wireless Sensor Network is one of the effective technologies of the ubiquitous computing environment [15]. So, through which the Ordenz.et.al [11] were able to collect the dataset of two individuals of their ADLs using binary sensors which are

*E-mail address: Siddhaling @ dubai.bits-Pilani.ac.in ISSN: $1791-2377$ @ 2020 School of Science, IHU. All rights reserved. doi:10.25103/iestr.132.25 tagged as most promising elements in solving the ADLs recognition problems. So much of work is in progress pertaining to the inclusion of context-awareness in database and database modeling tools. C.Bolchini et.al have discussed interpolation of the concepts mobility and context awareness in small database design through a comprehensive methodology [2]. Cristiana Bolchini et.al has projected on logical and physical design phases of smart card databases [3]. Here complete methodology of VSDB is been discussed which mainly includes Logistic phase where physical data structure, optimizing the cost and power consumption of the Flash memory been highlighted. ADLs using binary sensors dataset is been used for the conceptual and logical design of the context database design. Smart system dataset captured by Ordenza et.al using binary sensors through wireless sensor network is been used for database design. It has been elicited in ubiquitous computing environment associated with context-aware computing so, we envisioned this context dimensions of dataset as appropriate.

In Relational Database Management System (RDBMS) which is a static database where it shows no interest to represent and store the different contexts of the dynamic databases which are distributed in nature. Ubiquitous computing with context awareness facility using wireless sensor network generates data whenever sensors sense the users and it requires the apt data model to represent and store the data. So, it requires a unique approach to achieve the latter scenarios. In this paper using ADLs dataset which was collected using binary sensors through WSN been effectively used to solve the above-mentioned problem. With Activities of daily living application conceptual and logical design of context-aware database been achieved $[2,3]$. In conceptual 
design the paper defines and describes the context dimension tree which is the context data model used to represent different dimensions of ADLs application [1], context-aware use case diagram which depicts functional requirements [9] and different contexts of the application and finally Context Entity Attribute Relationship (CEAR) diagram which shows relationship between different contexts. This research work is unique in its nature pertaining to the database modeling part where context-aware entity relationship diagram [1], context-aware use case the diagram $[6,7,8]$ and the context dimension tree for view design are been designed effectively [10]. By which we can achieve elicitation of contextawareness requirements and functional requirement efficiently [9]. By utilizing context data with different dimensions like different users, time is taken, location, activity, place, etc. captured by Ordenza et.al [2], database is been created in RDBMS environment [16]. Few simple queries are written mathematically using relational algebra and executed to retrieve different contexts of the users using SQL and represented queries using query visualization Wolfgang [31]. Rule-based classification is required for decision making and ensemble learning is required for group decision making [27] [28]. Fuzzy decision making is iterative and interactive in decision making process [30].

Nearly all rule-based classifiers are either hinge on the 'divide and conquer' or the 'separate and conquer' rule induction approaches [21]. 'separate and conquer' stationed classifiers produce a group of if...then classification rules, such as the Prism family of algorithms [22,23,24] Prism takes an input as a file of ordered set of attributes values and generates rules [19]. In this research work, we have introduced attribute value block which is used to represent attribute value in query visualization which is a new concept [31]. It's a new kind of attempt to show that if have context data it can be used to create context database and user's context can be found by querying. Also context data can be reasoned by framing rules using Prism rule-based algorithm [20].The paper is structured as follows: In section 2 ADLs context database architecture is explained, section 3 steps in PRISM rule-based algorithm, section 4 context database design methodology, section 5 context dimension tree, section 6 conceptual phase, section 7 logical phase, section 8 experimental setup and results, section 9 Prism rule-based and in section 10 conclusion is been described.

\section{Adls Context Database Architecture Review Stage}

The context database architecture is as shown in fig.1. When users sensed by the sensors (PIR, Magnetic, Flush, Pressure, Electric ) which are been installed in different locations (bed, cabinet, basin, toilet, shower, fridge, cupboard, toaster, microwave, basin, main door, seat, cooktop) of living room places (entrance, living, bedroom, bathroom, kitchen) data will be stored in the data collection boards.

Collected ADLs dataset is been used in RDBMS environment to extract different contexts of the users with the help of relational algebra mathematically and SQL queries.

\section{Steps in Prism Rule based algorithm}

Prism algorithm is a rule-based algorithm which cajoles modular rules using separate and conquers approach [20]. This method gives rise to if-then rule. The aim is to induce modular assortment rules directly from the training set [18]. The algorithm which is shown above in the fig. 2 speculates that all the attributes are categorical. When there are continuous attributes first it has to be converted into a categorical attribute. Here when the rules are generated each rule belongs to individual class. Each rule is produced term by term with each term of the form 'attribute = value' Input is a training dataset with $m$ classes $C_{j} j=1,2,3,4,5 \ldots . m$ output generated rules for all the classes.

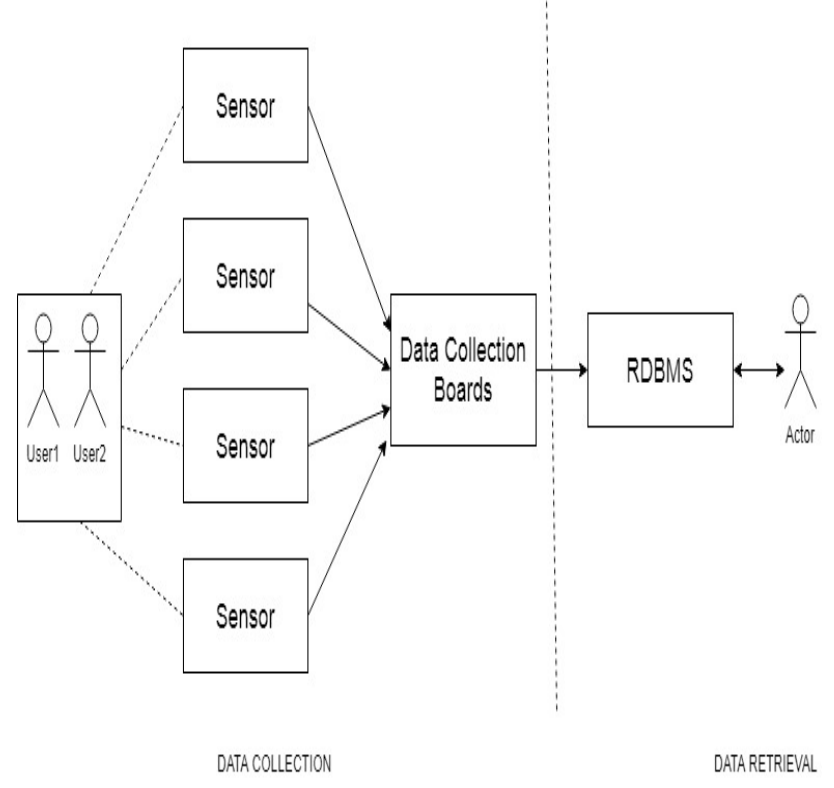

Fig. 1. ADLs Context Database Architecture

Step1: For each class $\mathrm{C}$ initialize $\mathrm{E}$ to the complete instance set.

Step2: while E contains instances with class $C$.

Step3: Create empty rule $\mathrm{R}$ if $\mathrm{X}$ then $\mathrm{C}$.

Step4: Until R is perfect (or no more attributes) for each attribute $A$ not in $R$, and each value $v$.

Step5: Consider adding $A=v$ to $R$.

Step6: Select A and v to maximize accuracy of $\mathrm{p} / \mathrm{t}$.

Step7: Add $A=v$ to R.

Step8: Remove instances covered by R from E.

Step9: Returns a list of all possible values $\left(\mathrm{v}_{1} \ldots \ldots \mathrm{v}_{\mathrm{n}}\right)$ of a given attribute.

Step10: Returns a list of all unique values. $\left(u_{1} \ldots \ldots u v_{n}\right)$ of a list.

Step 11: Returns list of attributes $\left(A_{1} \ldots \ldots . . A_{n}\right)$ not present in the rules.

Step12: Debugging function printing the set of rules $\left(R_{1} \ldots \ldots R_{n}\right.$ in English.

PRISM takes training set as input in the form of a file with ordered set of attribute values. Information about the attribute (ex: Userid, Start_time, End_time, location, activity, type, place.... etc.) will be the individual rules for each class [19]. Context database is a zestful domain so with zing, laconic data is used. In which data is imbued as input and the prism algorithm has exuded the output in the form of reasoning. It is apparent that to solve the problem mentioned Algorithm required data and it is touted for that. When data is imbued the system is slated with sundry elements to reason it by executing rules. It exudes the data at different context of the user. It will be instrumental in finding the contexts of the users. 


\section{Context Database Design Methodology}

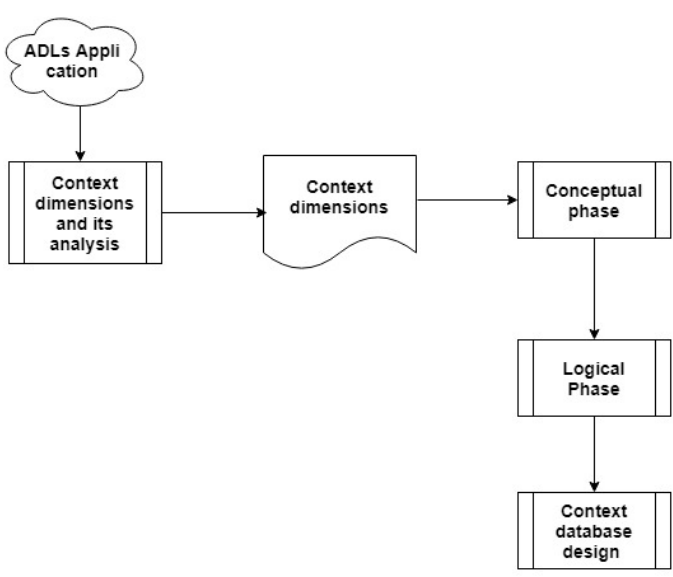

Fig. 2 Context database methodology

According to Dey and Abowd, the context can be defined as "Any information that can be used to characterize the situation of an entity" [25]. So, according to the requirements of the ADLs application chosen, different dimensions are been identified and thoroughly analyzed of their importance in our research work as shown in fig.2. Context dimensions chosen are role, interest topic, situation, time, interface, place, location and these dimensions are been represented meaningfully using context dimension tree [3, 4]. The conceptual phase is aided by CEAR diagram [1] and logical phase with tables comprising of ADLs dataset values [5]. It is called a context database because we have enumerated different contexts of the users using ADLs application with data reasoning capability.

\section{Context dimension tree}

Context-aware database design or any database design should be seen from two perspectives. First is conceptual framework and secondly data view design. So, in this paper first perspective has been achieved using context-aware use case diagram and context-aware entity attribute relation diagram. To achieve second perspective, need unique approach this is named as context dimension tree. According to [17] context data model can be defined as; it is an array of ambient dimensions used to model the perspectives from which the data are viewed and to derive the context as one or more instantiations of such an array.

The application considered in this paper is ADLs. When the main parameters of the dataset are represented using CDT its structure is conceptualized as shown below.

The important elements of this CDT are context dimensions as shown in fig.3. The different paradigm of the particular context can be enumerated as follows in table 1 and table 2 .

Table 1. Context \& Context Dimension Values

\begin{tabular}{l|l|l}
\hline Sl.no & $\begin{array}{l}\text { Context } \\
\text { dimensions }\end{array}$ & Context dimension values \\
\hline 1 & Role & User_1, User_2
\end{tabular}

Leaving, Toileting, howering, Sleeping, Breakfast, Lunch, Dinner, Snack, Spare time /TV, Grooming.

Routine(35days)

Today/Term.

Sensors (PIR, Magnetic, Flush,

Pressure, Electric)

Entrance, Living, Bedroom,

Bathroom, Kitchen.

Bed, Cabinet, Basin, Toilet,

Shower, Fridge, Cupboard, Toaster, Microwave, Basin, Main door, Seat, cooktop.

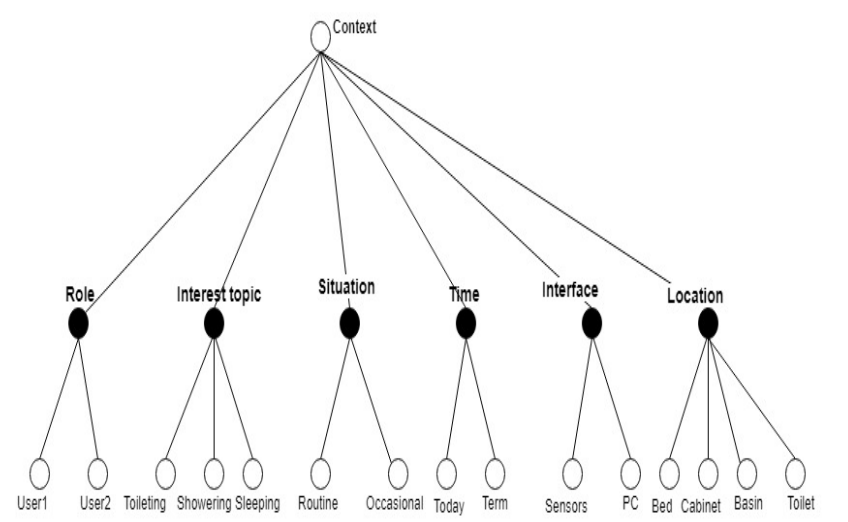

Fig. 3 Context dimension tree

Table 2. Context Dimensions Descriptions

\begin{tabular}{|c|c|c|}
\hline SL.No & $\begin{array}{l}\text { Context } \\
\text { dimensions }\end{array}$ & Description \\
\hline 1 & Role: & $\begin{array}{l}\text { In ADLs application, dataset } \\
\text { user } 1 \text { and user } 2 \text { are the main } \\
\text { entities whose activities for } \\
35 \text { total days is been captured } \\
\text { to monitor their health status. }\end{array}$ \\
\hline 2 & Interest topic & $\begin{array}{l}\text { The different activities } \\
\text { performed by the two users } \\
\text { during their stay in the living } \\
\text { room. }\end{array}$ \\
\hline 3 & Situation & $\begin{array}{l}\text { This means whether the } \\
\text { activities were held } \\
\text { continuously i.e. routine } \\
\text { activities or occasionally } \\
\text { participated. In our } \\
\text { application, it is a routine } \\
\text { activity. }\end{array}$ \\
\hline 4 & Time & $\begin{array}{l}\text { Time taken for particular } \\
\text { activities is absolute or } \\
\text { relative. In this paper } \\
\text { absolute time is been } \\
\text { considered. They are start } \\
\text { time and end time of different } \\
\text { activities. }\end{array}$ \\
\hline 5 & Interface & $\begin{array}{l}\text { Through which information } \\
\text { been collected either human } \\
\text { being or machine. In } \\
\text { particular case information } \\
\text { collected with the help of } \\
\text { sensors }\end{array}$ \\
\hline 6 & Place & This is the new dimension \\
\hline
\end{tabular}




\begin{tabular}{l|l|l} 
& $\begin{array}{l}\text { which we have added to the } \\
\text { CDT. There is a difference } \\
\text { between place and location, } \\
\text { Place comprises many } \\
\text { locations. } \\
\text { The point at which various } \\
\text { activities happened is called } \\
\text { location. }\end{array}$ \\
\hline
\end{tabular}

\section{Conceptual phase}

Context-aware applications gather data by sensing with the help of context devices called sensors to take decisions on change of contexts of users. These cognitive behaviors of the systems endorse them as special and of enticing nature. In software engineering use case diagrams are used to collect the functional requirements of the software applications.

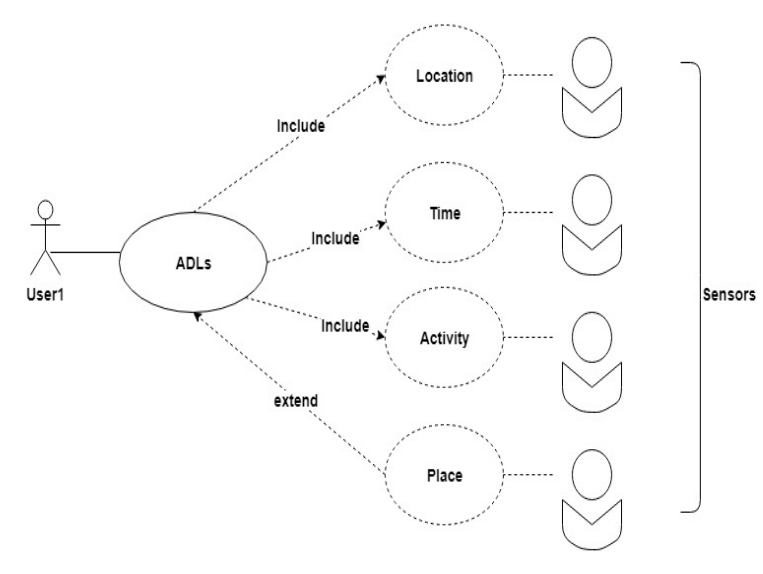

Fig. 4. Context-aware use case diagram

But similar things cannot be applied for context-aware applications here we need a concept which envisages and represents functional requirements and contexts of the applications. Such shortcomings can be refrained by contextaware use case diagram as shown in fig 4. Use context diagram comprises of users as actors, use case, use context, associations and Context devices. Users are the entities that will be exposed to context-aware applications. Use cases are useful to amass the functional requirements. Associations are included, exclude and utilize. Utilize relationship between use cases and use context means demeanor limited by the use case depends upon context information described by the use context. Context devices are different types of sensors, association between use case and use context are formed using dashed lines.

\section{CEAR Diagram}

CEAR diagram means context entity attribute relationship diagram it is used for a pictorial representation of relationship between context entity and its various attributes. It is an extension of ER diagram. It explains logical and physical structure of ADLS smart system. Logical structure connotes the relationship between entities, attributes, and types of attributes. Physical structure gives an idea about different types of sensors installed and devices used to capture the context data. Overall CEAR diagram depicts the conceptual design of ADLs smart system in ubiquitous computing environment as shown in fig 5 .

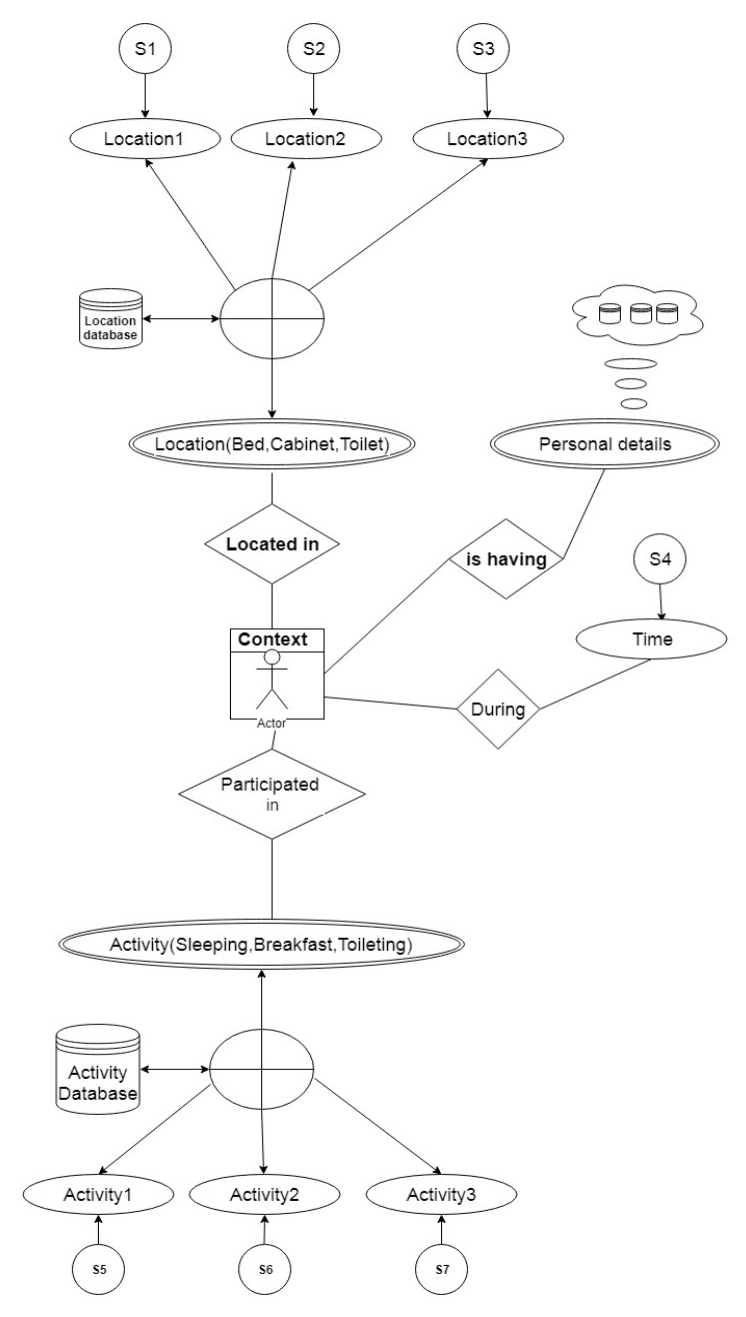

Fig. 5. CEAR diagram

\section{Logical Phase}

"Logical database design is the process of deciding how to arrange the attributes of the entities in a given business environment into database structures, such as the tables of a relational database" MARK L. GILLENSON [26]. The purpose of the logical database design is to produce wellstructured tables that properly contemplate the business environment. Logical design allows one to analyze the data itself as shown in the fig.7

1. ADLs1(UserID, Start time End time,Activity)

2. ADLs1Sensor (UserID, Start time End time, Location, type, Place)

Schema diagram is used to represent entities and their relationships. Here two entities and their attributes are being pictorially represented using the primary key and foreign key as shown in fig 6 .

\section{A. Physical Database}

The physical database is the transfigure of data model into physical data structure by ensuring data redundancies using data integrity. 


\section{B. Schema diagram}

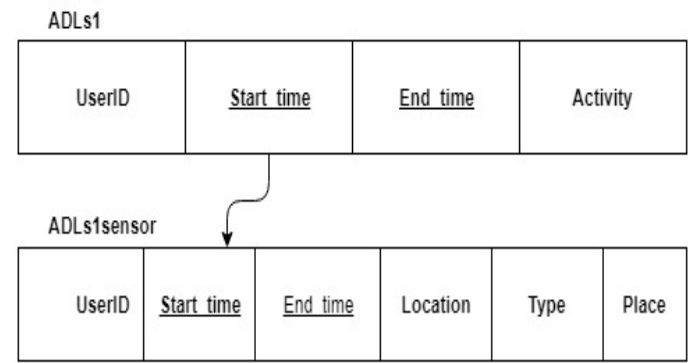

Fig. 6. Schema diagram

Table 3. ADLs1

\begin{tabular}{l|l|l|l}
\hline UserID & Start_time & End_time & Activity \\
\hline User1 & $2011-11-28$ 02:27:59 & $2011-11-28$ 10:18:11 & Sleeping \\
User1 & $2011-11-28$ 10:21:24 & $2011-11-28$ 10:23:36 & Toileting \\
User2 & $2012-11-12$ 01:54:00 & $2012-11-12$ 09:31:59 & Sleeping \\
User2 & $2012-11-12$ 10:53:00 & $2012-11-12$ 10:53:59 & Toileting \\
\hline
\end{tabular}

Table 4. ADLS1sensor

\begin{tabular}{l|l|l|l|l|l}
\hline UserID & Start time & End time & Location & Place & Type \\
\hline User1 & $2011-11-$ & $2011-11-$ & Bed & Bedroom & Pressure \\
& 28 & 28 & & & \\
& $02: 27: 59$ & $10: 18: 11$ & & & \\
User1 & $2011-11-$ & $2011-11-$ & Cabinet & Bathroom & Magnetic \\
& 28 & 28 & & & \\
& $10: 21: 24$ & $10: 23: 36$ & & & \\
User2 & $2012-11-$ & $2012-11-$ & Bed & Bedroom & Pressure \\
& 12 & 12 & & & \\
& $01: 54: 00$ & $09: 31: 59$ & & & \\
User2 & $2012-11-$ & $2012-11-$ & Cabinet & Bathroom & Magnetic \\
& 12 & 12 & & & \\
& $10: 53: 00$ & $10: 53: 59$ & & & \\
\hline
\end{tabular}

C. Relational Algebra

Relational algebra is a procedural query language which takes exemplar of relation as input and gives exemplar of relation as output. It uses unary and binary operators to perform a query.

Usage of select operation and Project operation:

Query1: Retrieve the user1 context pertaining to the activity.

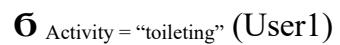

Query2: Retrieve start time and end time of User1 context.

$\boldsymbol{\pi}$ Start time, End time (User1)

Query3: Retrieve the context details of user1 when he performs breakfast.

$\boldsymbol{\pi}$ Place, Location, Start time, End time $\left(\boldsymbol{6}_{\text {Activity }}=\right.$ "breakfast" $($ User 1$\left.)\right)$

Query4: Retrieve the context details Location; place at which users have their breakfast using Cartesian product.

$$
\text { Temp } \leftarrow \boldsymbol{6}_{\text {Activity }=\text { "breakfast" }}(\text { User } 1)
$$

Temp $1 \leftarrow \boldsymbol{\pi}$ UserID, Start time, End time (Temp)

Location_Temp $\leftarrow$ Temp 1 X User2

Final_Result $\leftarrow \boldsymbol{\pi}_{\text {Location, place (Location) }}$

\section{Tuple Calculus}

We can find tuples for which a predicate is true in the tuple relational calculus, the calculus is dependent on the use of tuple variables [32]. In tuple calculus a query can be expressed as $\{\mathbf{t} \mid \mathbf{P}(\mathbf{t})\}$, where $t$ is resulting tuples, $\mathbf{P}(\mathbf{t})$ is known as predicate and these are the conditions that are used to fetch t. contexts where user is sleeping can be represented and queried as follows.

i. Set of all tuples $t$ such that $t$ belongs to ADLS1 and Activity is sleeping

$\{\mathbf{t} \mid \mathbf{t} \in \operatorname{ADLs} 1 \Lambda \mathrm{t}[$ Activity $]=$ Sleeping $\}$

ii. Set of all tuples $t$ such that there exist A belongs to the relation ADLS1. Starting time And Activity is Sleeping

$\left\{\mathrm{t} \mid \exists \mathrm{A} \in \mathrm{ADLS} 1\left(\mathrm{t}\left[\mathrm{St} \_\right.\right.\right.$time $]=\mathrm{A}\left[\mathrm{St} \_\right.$time $] \boldsymbol{\Lambda} \mathrm{A}[$ Activity $]=$ Sleeping)\}

\section{Experimental Setup and Results}

The real dataset captured by the binary sensors [11] in a ubiquitous environment is been used to create context database using oracle software in RDBMS environment. Database been created using SQL Data definition language, even data manipulation language been used on a certain occasion, simple queries were written using relational algebra and SQL. SQL queries are executed to retrieve the different contexts of the users.

A. Dataset

Table 5. Ordenza_activities of the daily living

\begin{tabular}{l|l|l|l}
\hline $\begin{array}{l}\text { User } \\
\text { ID }\end{array}$ & Start Time & End Time & Activity \\
\hline User1 & $\begin{array}{l}2011-11-28 \\
02: 27: 59\end{array}$ & $2011-11-28$ 10:18:11 & Sleeping \\
User1 & $\begin{array}{l}2011-11-28 \\
10: 21: 24\end{array}$ & $2011-11-2810: 23: 36$ & Toileting \\
User1 & $\begin{array}{l}2011-11-28 \\
10: 25: 44\end{array}$ & $2011-11-2810: 33: 00$ & Showering \\
User1 & $\begin{array}{l}2011-11-28 \\
10: 34: 23\end{array}$ & $2011-11-2810: 43: 00$ & Breakfast \\
User1 & $\begin{array}{l}2011-11-28 \\
10: 49: 48\end{array}$ & $2011-11-2810: 51: 13$ & Grooming \\
User1 & $\begin{array}{l}2011-11-28 \\
10: 51: 4\end{array}$ & $2011-11-2813: 05: 07$ & Spare-time/TV \\
User1 & $\begin{array}{l}2011-11-28 \\
13: 06: 04\end{array}$ & $2011-11-2813: 06: 31$ & Toileting \\
User1 & $\begin{array}{l}2011-11-28 \\
13: 09: 31\end{array}$ & $2011-11-2813: 29: 09$ & Leaving \\
User1 & $\begin{array}{l}2011-11-28 \\
13: 38: 40\end{array}$ & $2011-11-2814: 21: 40$ & Spare_Time/TV \\
User1 & $2011-11-28$ & $2011-11-2814: 27: 07$ & Toileting \\
& $14: 22: 38$ & & \\
\hline
\end{tabular}

Table 6. Ordenza Data Recorded Using Wireless Sensor Network.

\begin{tabular}{c|l|l|l|l|l}
\hline $\begin{array}{c}\text { User } \\
\text { ID }\end{array}$ & $\begin{array}{c}\text { Start } \\
\text { Time }\end{array}$ & $\begin{array}{c}\text { End } \\
\text { Time }\end{array}$ & Location & TYPE & PLACE \\
\hline User1 & $\begin{array}{l}2011-11- \\
28\end{array}$ & $\begin{array}{l}2011-11- \\
28\end{array}$ & Bed & Pressure & Bedroom \\
& & & &
\end{tabular}




\begin{tabular}{|c|c|c|c|c|c|}
\hline & $02: 27: 59$ & $10: 18: 11$ & & & \\
\hline User1 & $\begin{array}{l}2011-11- \\
28 \\
10: 21: 24\end{array}$ & $\begin{array}{l}2011-11- \\
28 \\
10: 21: 31\end{array}$ & Cabinet & Magnetic & Bathroom \\
\hline User1 & $\begin{array}{l}2011-11- \\
28 \\
10: 21: 44\end{array}$ & $\begin{array}{l}2011-11- \\
28 \\
10: 23: 31\end{array}$ & Basin & PIR & Bathroom \\
\hline User1 & $\begin{array}{l}2011-11- \\
28 \\
10: 23: 02\end{array}$ & $\begin{array}{l}2011-11- \\
28 \\
10: 23: 36\end{array}$ & Toilet & Flush & Bathroom \\
\hline User1 & $\begin{array}{l}2011-11- \\
28 \\
10: 25: 44\end{array}$ & $\begin{array}{l}2011-11- \\
28 \\
10: 32: 06\end{array}$ & Shower & PIR & Bathroom \\
\hline User1 & $\begin{array}{l}2011-11- \\
28 \\
10: 34: 23\end{array}$ & $\begin{array}{l}2011-11- \\
28 \\
10: 34: 41\end{array}$ & Fridge & Magnetic & Kitchen \\
\hline User1 & $\begin{array}{l}2011-11- \\
28 \\
10: 34: 44\end{array}$ & $\begin{array}{l}2011-11- \\
28 \\
10: 37: 17\end{array}$ & Cupboard & Magnetic & Kitchen \\
\hline User1 & $\begin{array}{l}2011-11- \\
28 \\
10: 38: 00\end{array}$ & $\begin{array}{l}2011-11- \\
28 \\
10: 42: 41\end{array}$ & Toaster & Electric & Kitchen \\
\hline User 1 & $\begin{array}{l}2011-11- \\
28 \\
10: 38: 33\end{array}$ & $\begin{array}{l}2011-11- \\
28 \\
10: 38: 40\end{array}$ & Fridge & Magnetic & Kitchen \\
\hline User1 & $\begin{array}{l}2011-11- \\
28 \\
10: 41: 29\end{array}$ & $\begin{array}{l}2011-11- \\
28 \\
10: 41: 36\end{array}$ & Cupboard & Magnetic & kitchen \\
\hline
\end{tabular}

The raw data obtained by the binary sensors via wireless sensor networks are been manually labeled by Ordenza.et.al are been used in the context database design. Database design comprises of data modeling, database creation, querying using relational algebra and SQL. Dataset is also been used in PRISM algorithm to assert the facts, to frame the rules Using labeled binary sensor data, it was possible to create context database in Oracle 11.2.0 version, SQL plus software using 32-bit machine. We successfully represented few context dimensions like User id, Location and Time. These context dimension's data are used to create few relations and established relationship required for database design. Using prism rule base data reasoning was implemented successfully. The results are as shown below.

\section{B. SQL Queries (Data Definition Language)}

CREATE TABLE ADLs1(UserID VARCHAR (10), Start time DATETIME PRIMARY KEY, End time DATETIME PRIMARY KEY, Activity VARCHAR (20));

CREATE TABLE ADLs1Sensor (UserID CHAR (10), Start time DATETIME PRIMARY KEY, End_time DATETIME PRIMARY KEY,

Location VARCHAR (20),

Type VARCHAR (20),

Place VARCHAR (20),

Foreign key Start_time references ADLs1 (Start_time));

\section{Adls1 Insertions}

1. INSERT INTO ADLs1 VALUES ('User1', '201111-28 10:21:24', '2011-11-28 10:23:36', 'Toileting');

2. INSERT INTO ADLs1 VALUES ('User1', '201111-28 02:27:59', '2011-11-28 10:18:11', 'Sleeping');

\section{Simple Queries}

Retrieve the userl context pertaining to the activity.

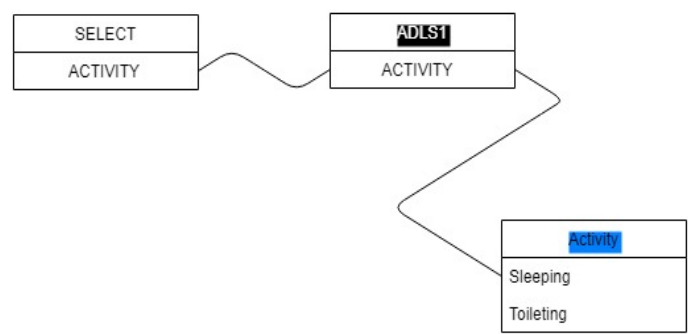

Fig. 7. User activity

\begin{tabular}{ll} 
Output & \\
\hline & Activity \\
\hline Toileting \\
Toileting
\end{tabular}

Retrieve start time and end time of User1 context.

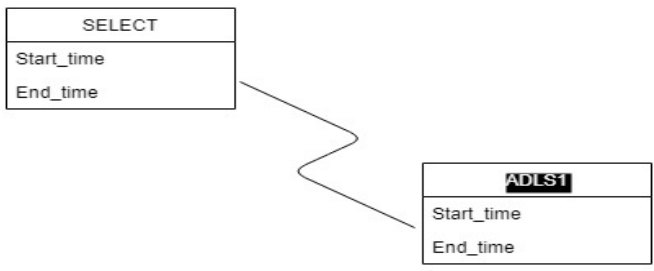

Fig. 8. Context time

\section{Output}

\begin{tabular}{l|l}
\hline Start_time & End_time \\
\hline $2011-11-2802: 27: 59$ & $2011-11-28 \quad 10: 18: 11$ \\
$2011-11-28 \quad 10: 21: 24$ & $2011-11-28 \quad 10: 23: 36$ \\
$2012-11-12 \quad 01: 54: 00$ & $2012-11-12 \quad 09: 31: 59$ \\
2012-11-12 10:53:00 & $2012-11-12 \quad 10: 53: 59$ \\
\hline
\end{tabular}

Retrieve the context details of user1 when he performs breakfast.

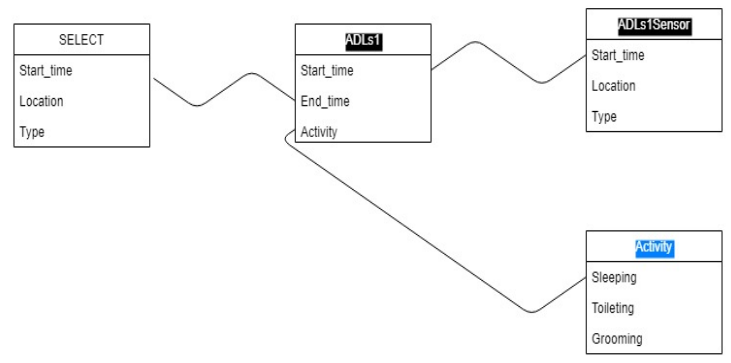

Fig. 9. Context activity

\begin{tabular}{l|l|c|l} 
Output & \multicolumn{3}{l}{ Type } \\
\hline Start_time & End_time & Location & Pypes \\
\hline $02: 27: 59$ & $2011-11-28$ & Bed & Pressure \\
$2012-11-12$ & $20: 18: 11$ & & \\
$01: 54: 00$ & $09: 31: 59$ & Bed & Pressure \\
& & & \\
\hline
\end{tabular}




\section{PRISM RULE-BASED}

\section{A. Input Data}

During initialization, accomplishment oodles the .arff file and converts it into an apt illustration. This is made by using the arff2skl () class defined in util2.py (self._cvt in code) the conversion of the dataset is done by calling the to dict () method by calling this method we decipher the dataset from the. arff format.

\begin{tabular}{l|l|l|l}
\hline$\underline{\text { UserID }}$ & Start_time & End_time & Activity \\
\hline User1 & $2011-11-28$ 02:27:59 & $2011-11-28$ & Sleeping \\
& & $10: 18: 11$ & \\
User1 & $2011-11-28$ 10:21:24 & $2011-11-28$ & Toileting \\
& & $10: 23: 36$ & \\
User2 & $2012-11-1201: 54: 00$ & $2012-11-12$ & Sleeping \\
& & $09: 31: 59$ & \\
User2 & $2012-11-1210: 53: 00$ & $2012-11-12$ & Toileting \\
& & $10: 53: 59$ & \\
\hline
\end{tabular}

\begin{tabular}{l|l|l|l|l|l}
\hline UserID & Start time & End time & Location & Place & Type \\
\hline User1 & $2011-11-28$ & $2011-11-28$ & Bed & Bedroom & Pressure \\
& $02: 27: 59$ & $10: 18: 11$ & & & \\
User1 & $2011-11-28$ & $2011-11-28$ & Cabinet & Bathroom & Magnetic \\
& $10: 21: 24$ & $10: 23: 36$ & & & \\
User2 & $2012-11-12$ & $2012-11-12$ & Bed & Bedroom & Pressure \\
& $01: 54: 00$ & $09: 31: 59$ & & & \\
User2 & $2012-11-12$ & $2012-11-12$ & Cabinet & Bathroom & Magnetic \\
& $10: 53: 00$ & $10: 53: 59$ & & & \\
\hline
\end{tabular}

B. Dataset Representation using List of Dictionaries Data $=$ [\{'USERID': 'user1', 'Start_time': '2011-11-28 02:27:59', 'End time': '2011-11-28 10:18:11', 'Location': 'Bed', 'type': 'Pressure', Place': 'Bedroom', 'Activity': 'sleeping'\}

\{'USERID': 'user2', 'Start_time': '2011-11-12 10:53:00', 'End_time': '2011-11-12 10:53:59', 'Location': 'cabinet', 'type': 'Magnetic', 'Place': 'Bathroom', 'Activity': 'toileting' $\}$.....

Other information such as: attributes and label are extracted from the metadata of the. arff these are obtained by:

Attributes = self._cvt.meta.names () \# ['USERID', 'start time'...]

Label $=$ attributes [-1] \# 'ADLs' (what we want to predict)

Classes $=$ Self._cvt.meta [Label] $[1] \#$

['Sleeping', 'toileting']

\section{A Rule is encoded as a List of One or More}

Predicates:

In the case of the ADSLs dataset, a rule for 'sleeping' class is:

'Place' = = 'bedroom' and

'Location' == 'bed' and

'Type' $==$ 'pressure'

Here the rule is encoded as a list of tuples of the form:

Rule $=$ [('place', 'bedroom'), ('location', 'bed'), ('type', 'pressure')]

\section{Output}

The output of Prism rule-based is the set of rules regressed by the fit () method of the prism class. The set of all rules are delineated as a list of dictionaries enumerated by all the possible classes.

In the case of ADLs dataset, the output is as shown below:

$\mathrm{R}=$ [\{'sleeping': [('place', 'bedroom'), ('location', 'bed'), ('type', 'pressure')]\},

\{'toileting': [('place', 'bathroom'), ('location', 'cabinet'), ('type', 'magnetic')]\} ...

Rule1: If a user is in the bedroom at location bed captured by pressure sensor

Then the user is sleeping.

Rule2: If a user is in the bathroom at location cabinet captured by magnetic sensor

Then the user is toileting.

From table 5 and Table 6 The data visualization of the dataset is as shown below.

Graphs are been plotted for ADLs dataset at different instances of both the users i.e. user1 and user2. All the attributes are taken into consideration to show the daily activities of the users graphically. From the graph we can make out both the users are active and they can perform most of the work themselves. Prism algorithm efficiently represents the dataset with set of rules.

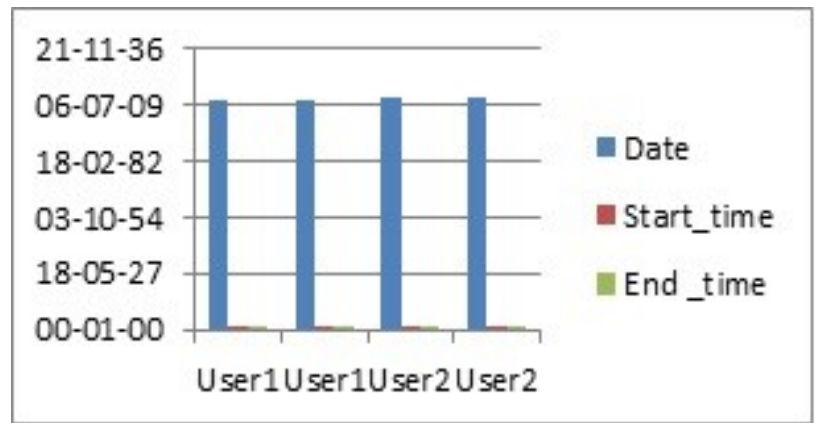

Fig. 10. User activities in particular dates

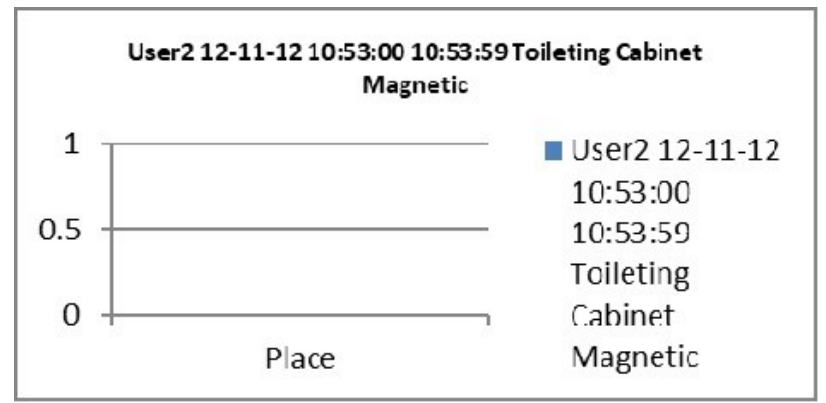

Fig 11. User 2 activity active scenarios

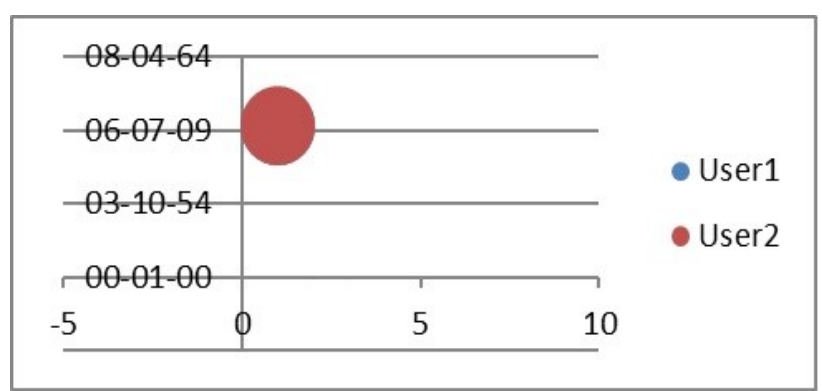

Fig. 12. user 1 and user 2 comparison 


\section{User2 12-11-12 10:53:00 10:53:59 Toileting}

(1) - User2 12-11-12...

Location Bed

\section{— User2 12-11-12 10:53:00 10:53:59 Toileting}

Fig. 13. User 2 contexts w.r.t location

The user activities and their ability to perform their work on their own are been represented using different contexts of the user1 and user2. It is pretty clear user2 marginally outclass userl and the situations are been diagrammatically represented in the fig.10, fig.11, fig.12 and fig.13. This graph depicts the various contexts of the users.

\section{Conclusion}

The data which has implicit meaning can be easily represented with the suitable data model, stored and retrieved efficiently. The data which is captured by the sensors will be unstructured so rendition and other operations will become onerous. Sensor generated data includes different contexts of the different users which needs a suitable context database to store and perform required operations. Using RDBMS, we have assayed storage of unstructured data. Here we have made an attempt to showcase the data storage and retrieval in a similar fashion as for the structured data and queries are written in tuple calculus environment. Context database design encompasses many stages starting from, context view design using context dimension tree, conceptual design using CEAR diagram and context-aware user case diagram. Context view design is engineered using context dimension tree which unravels different dimensions of the user. Query visualization and data reasoning are the topics where most of the authors have not paid heed. We have used data visualization to represent query pictorially and real-time data generated by binary sensors it is ADLs dataset. Our novel contribution is implementation of PRISM rule-based algorithm using ADLs data for rule generation and introduced attribute value block in the query visualization part. Since we have used small set of data it can be easily equipped but for colossal amount of data NO-SQL is apropos.

This is an Open Access article distributed under the terms of the Creative Commons Attribution License

\section{References}

1. Shivakumar.C, Dr. Siddhaling Urolagin," Context Data Representation using Context-Aware. Model in Ubiquitous Computing Scenario", May 2017, International Journal of Innovations \& Advancement in Computer Science, ISSN 2347 8616 Volume 6, Issue 5.

2. C.Bolchini, F.A.Schreiber L.Tanca "A Methodology for Very small Database Design", 29th October 2004, Politecnico di Milano, Dipartmento di Elettronica e Informazione, P.zza L. Da Vinci, 3220133 Milano - Italy.

3. Cristinana Bolchini, Fabio Salice, Fabio A. Schreiber and Letizia Tanca" Logical and Physical Design Issues for Smart Card Databases", ACM Transactions on Information Systems, Vol.21, No.3, July 2003, Pages 254-285

4. C.Bolchini, F.A.Schreiber L.Tanca " A Context-Aware Methodology for Very small Design”, SIGMOD Record, Vol.33, No.1, March 2004.

5. Ordóñez, F.J.; de Toledo, P.; Sanchis, A. “Activity Recognition Using Hybrid Generative Discriminative Models on Home, Environments Using Binary Sensors", 2013, 13, 5460-5477

6. Ahmed Al-alshuhai, François Siewe," An Extension of the Use Case Diagram to Model Context-Aware Applications", SAI Intelligent Systems Conference 2015 IEEE, November 10 11, 2015, London, UK, 978-1-4673-7606-8/15

7. Ahmed Al-alshuhai * and François Siewe ** Software Technology Research Laboratory, De Montfort University, Leicester, United Kingdm" An Extension of Class Diagram to Model the Structure of Context-AwareSystems"*p07143453@myemail.dmu.ac.uk **siewe@odmu.ac.uk

8. Mohamed-Salah Benselim1 and Hassina Seridi-Bouchelaghem2 ,1Department of Management Science, University of "08 Mai 45", Algeria, 2Department of Computer Science University of Badji Mokhtar, Algeria," Towards A UML Profile for ContextAwareness Domain", The International Arab Journal of Information Technology, Vol. 14, No. 2, 2017

9. J.Choi and Y.Lee. "Use-Case Driven Requirements Analysis for Context-aware Systems".In the future generation information technology conference, springer, 2005, Australia

10. C.Bolchini, C.A. Curino, E.Quintarelli, F.A. Schreiber and L.Tanca "Context information for knowledge shaping", Int.J. Web Engineering and Technology, Vol. 5, No.1, 2009.
11. Ordóñez, F.J.; de Toledo, P.; Sanchis, A. Activity Recognition Using Hybrid Generative/ Discriminative Models on Home Environments Using Binary Sensors. Sensors 2013, 13, 5460-5477.

12. Zola, I.K. "Living at Home: The Convergence of Aging and Disability"." In Staying Put- Adapting the Places Instead of the People"; Lanspery, S., Hyde, J., Eds.; Baywood. Publishing: Amityville, NY, USA, 1997; pp. 25-39

13. United Nations Department of Economic and Social Affairs, 2008

14. Subhojit Dey, Devaki Nambiar, J. K. Lakshmi, Kabir Sheikh, and K. Srinath Reddy," Health of the Elderly in India: Challenges of Access and Affordability".

15. Corchado, J.M.; Bajo, J.; Tapia, D.I.; Abraham, A. Using heterogeneous wireless sensor networks in a telemonitoring system for healthcare. Trans. Inf. Tech Biomed. 2010, 14, 234-240.

16. Ramez Elmasri university of texas, Shamakant B. Navathe, Georgia Institute of Technology "Fundamentals of Database Systems", $7^{\text {th }}$ edition, ISBN 978-93-325-8270-5.

17. Anind K. Dey and Gregory D. Abowed, "Towards a Better Understanding of Context and Context-Awareness, Georgia Institute of Technology, Atlanta, GA, USA 30332-0280 \{anind, abowd\}@cc.gatech.edu.

18. Ishrat Nahar Farhana, A.H.M. Sajedul Hoque et.al, "Building a classifier employing prism algorithm with fuzzy logic", Department of Computer Science and Engineering, University of Chittagong, Chittagong, Bangladesh, IJDKP Vol.7, No.2, March 2017

19. Jadzia Cendrowska c/o the faculty of mathematics, The open university, Walton Hall, Milton Keynes, Mk76AA, U.K, int J.Man. Machine studies (1987)27, 349-370

20. Frederic Stahl and Max Bramer, "Random Prism: A Noise Tolerant Alternative to Random Forests" Stahl, F. and Bramer, M. (2014) Random Prism: a noise-tolerant alternative to Random Forests. Expert Systems, 31(5). pp. 411420. ISSN 14680394 doi.

21. Witten, I.H., Frank, E.” Data Mining: Practical Machine Learning Tools and Techniques: Practical Machine Learning Tools and Techniques" The Morgan Kaufmann Series in Data Management Systems. Elsevier Science, Amsterdam (2011).

22. Bramer, M., Macintosh, A., Coenen, F "Automatic induction of classification rules from examples using N-Prism”, Research and Development in Intelligent Systems XVI, pp. 99-121. SpringerVerlag, London (2000). 
23. Bramer, M.A, Musen, M.A., Neumann, B., Studer, R. (eds.) an information-theoretic approach to the pre-pruning of classification rules. Intelligent Information Processing. IFIP vol. 93, pp. 201-212. Springer, Boston (2002).

24. Cendrowska, J. Man Mach. Stud. "PRISM: an algorithm for inducing modular rules" 27(4), 349-370 (1987).

25. Anind K.Dey and Gregory D.Abowd. "Towards a Better Understanding of Context and Context-Awareness", George Institute of Technology, Atlanta, GA, USA 30332-0280 \{anind, abowd\}@cc.gatech.edu

26. MARK L. GILLENSON, "Fundamentals of Database Management Systems" Second Edition.

27. John Garmany, Jeff Walker, Terry Clark, "Logical Database Design Principles", Auerbach publications, Taylor and Francis group
28. Cendrowska, "PRISM: an algorithm for inducing modular rules", International Journal of Man-Machine Studies 27, 349-370 (1987).

29. Liu H., Gegov "A Collaborative Decision Making by Ensemble Rule-Based Classification Systems" (2015)

30. Pedrycz W., Chen SM (eds) "Granular Computing and DecisionMaking (Studies in BigData), vol 10, Springer, Cham

31. Wolfgang Gatterbauer, "Databases will Visualize Queries too", University of Washington, Seattle.

32. Bagai, Rajiv. "Tuple relational calculus for paraconsistent databases." In Advances in Artificial Intelligence, pp. 409-416. Springer, Berlin, Heidelberg, 2000. 